\title{
Estudio de la anatomía sagital de la pelvis de pacientes con dolor sacroilíaco en el ámbito laboral
}

\author{
Guillermo A. Ricciardi, Ignacio G. Garfinkel, Gabriel G. Carrioli, Daniel O. Ricciardi \\ Equipo de Columna, Centro Médico Integral Fitz Roy, Ciudad Autónoma de Buenos Aires, Argentina
}

\begin{abstract}
RESUMEN
Introducción: La articulación sacroilíaca es el origen del dolor en el 10-25\% de los pacientes con lumbalgia mecánica. Si no hay traumatismos, tumores, enfermedades metabólicas ni colagenopatías, su papel en la fisiopatología del dolor lumbar aún no está completamente dilucidado. El objetivo fue estudiar la anatomía sagital de la pelvis a través de la medición de la incidencia pélvica en pacientes con dolor sacroilíaco en el ámbito laboral frente a personas asintomáticas. Materiales y Métodos: Estudio analítico, observacional, retrospectivo de pacientes con dolor sacroilíaco diagnosticados mediante un bloqueo guiado por tomografía computarizada en un centro de patología laboral, entre enero de 2015 y diciembre de 2018. El grupo de comparación fue una muestra aleatoria de personas sin enfermedad de la pelvis o lumbosacra estudiados con tomografía computarizada de pelvis y columna durante el mismo período. Resultados: La incidencia pélvica media en pacientes con dolor sacroilíaco fue de 52,57 (DE 9) y 46,52 (DE 9) en el grupo de personas sanas. La diferencia fue estadísticamente significativa con valores mayores de incidencia pélvica en pacientes con dolor sacroilíaco ( $\mathrm{p}$, 042). Conclusión: Los pacientes con dolor sacroilíaco presentaron una mayor incidencia pélvica que las personas sanas, con una diferencia estadísticamente significativa en nuestro estudio.
\end{abstract}

Palabras clave: Incidencia pélvica; síndrome de dolor sacroilíaco; anatomía sagital.

Nivel de Evidencia: IV

\section{Study of the Sagittal Anatomy of the Pelvis in Workers With Sacroiliac Pain}

\section{ABSTRACT}

Introduction: The sacroiliac joint causes pain in 10 to $25 \%$ of patients with mechanical low back pain or symptoms radiating to the lower limbs. In the absence of trauma, metabolic disease, and collagen disease, its role in the pathophysiology of low back pain is not yet fully understood. The objective is to study the sagittal pelvic anatomy through the measurement of pelvic incidence $(\mathrm{PI})$ in patients with sacroiliac pain in the workplace compared to asymptomatic patients. Materials and Methods: A retrospective observational analytical study of adult patients with sacroiliac pain diagnosed with CT-guided block in an occupational pathology center during January 2015 and December 2018. The comparison group was a random sample of patients without pelvic or lumbosacral pathology studied with CT of the pelvis and spine during the same period. Results: The mean PI in patients with sacroiliac pain was 52.57 (SD 9) and 46.52 (SD 9) in the group of healthy patients. The difference was statistically significant with the higher $\mathrm{PI}$ values in patients with sacroiliac pain ( $\mathrm{p}$ 0.042). Conclusion: Patients with sacroiliac pain had a higher pelvic incidence than healthy patients, with a statistically significant difference in our study.

Key words: Pelvic incidence; sacroiliac pain; sagittal anatomy.

Level of Evidence: IV

\section{INTRODUCCIÓN}

La articulación sacroilíaca (ASI) es el origen del dolor en el 10-25\% de los pacientes con lumbalgia mecánica o síntomas irradiados a los miembros inferiores. ${ }^{1}$ Si no hay traumatismos, tumores, enfermedades metabólicas ni colagenopatías, su papel en la fisiopatología del dolor lumbar aún no está completamente dilucidado. ${ }^{2}$

Recibido el 22-5-2020. Aceptado luego de la evaluación el 29-10-2020 • Dr. GUILLERMO A. RICCIARDI • guillermoricciardi@gmail.com ID https://orcid.org/0000-0002-6959-9301 Cómo citar este artículo: Ricciardi GA, Garfinkel IG, Carrioli GG, Ricciardi DO. Estudio de la anatomía sagital de la pelvis de pacientes con dolor sacroilíaco en el ámbito laboral. Rev Asoc Argent Ortop Traumatol 2021;86(6):747-754. https://doi.org/10.15417/issn. 1852-7434.2021.86.6.1127 
La ASI tiene una movilidad limitada, quizás porque tiene características sinoviales solo en dos tercios. El resto tiene fibrocartílago y un conjunto intrincado de conexiones ligamentarias que le otorgan estabilidad intrínseca. La cápsula posterior rudimentaria es reforzada por fuertes ligamentos extrínsecos. Es razonable entonces que su función principal sea otorgar estabilidad y permitir la transmisión de las cargas del tronco a los miembros inferiores. ${ }^{3}$

La incidencia pélvica (IP), ángulo descrito por Duval-Beaupère, determina la posición del platillo superior de S1 y su relación con el centro de rotación de ambas caderas. ${ }^{4}$ Única e individual, otorga información sobre la anatomía sagital de la pelvis. Es variable con el crecimiento y estable en la madurez esquelética. La invariabilidad de este ángulo en el adulto está sujeta a la estabilidad de la unión sacroilíaca. ${ }^{4,5} \mathrm{La}$ IP promedio es $55^{\circ} \pm 10^{\circ}$ con un amplio rango normal $\left(\right.$ de $35^{\circ}$ a $\left.85^{\circ}\right) .^{6}$

Los pacientes con IP muy bajas tienen un eje anteroposterior pélvico corto configurando una "pelvis vertical", las cabezas femorales se ubican debajo del platillo superior de S1. En cambio, las IP muy elevadas con un eje anteroposterior largo configuran una pelvis horizontal, con cabezas femorales situadas por delante del platillo superior de $\mathrm{S} 1 .^{7}$

El paciente con una IP baja tiene una menor reserva de retroversión pélvica en oposición a aquellos con una IP muy elevada. La retroversión pélvica, mecanismo compensador clave en la adaptación a la postura de pie del ser humano, depende de la movilidad de las caderas, la acción muscular de los glúteos y músculos extensores de la columna que permitirán extender las caderas y horizontalizar el platillo superior del sacro, músculos que intervienen en la movilidad sacroilíaca junto al piramidal y bíceps femoral. ${ }^{3-9}$

Los autores planteamos la siguiente pregunta de investigación: ¿existen diferencias en la anatomía sagital de la pelvis, según la medición de la IP, en pacientes con dolor sacroilíaco y personas asintomáticas?

El objetivo fue estudiar la IP en pacientes con dolor sacroilíaco en el ámbito laboral comparándolos con personas asintomáticas. El objetivo secundario fue estudiar el valor de la IP en los pacientes sintomáticos, en función del tiempo de tratamiento hasta la resolución de los síntomas ( $<4$ semanas o $>4$ semanas).

\section{MATERIALES Y MÉTODOS}

Estudio retrospectivo, analítico observacional de pacientes adultos con dolor sacroilíaco diagnosticados con bloqueo sacroilíaco guiado por tomografía computarizada (TC) en un centro de derivación de patología laboral, entre enero de 2015 y diciembre de 2018. Se consideró como grupo de comparación una muestra aleatoria de personas sin enfermedad pélvica o lumbosacra evaluadas en nuestro centro mediante TC de pelvis y columna durante el mismo período.

Los criterios de inclusión fueron pacientes evaluados con TC de columna lumbosacra y pelvis, con escanograma (scout view) de perfil que permita visualizar la región lumbosacra y ambas caderas. Los criterios de exclusión fueron: 1) enfermedad de la pelvis y ambas caderas, 2) síndrome sacroilíaco secundario a fusión lumbosacra, deformidad vertebral, colagenopatías, tumores, infecciones y uso de ortesis toracolumbar, 3) imagen radiográfica de sacroileítis avanzada (grados 3 y 4 de la clasificación modificada de Nueva York), 4) trauma de pelvis reciente de alta energía (<1 año), 5) múltiples siniestros laborales previos (2 o más siniestros diferentes en un año), 6) historia clínica incompleta o estudios técnicamente inadecuados para la medición.

Se agrupó a los pacientes tratados con bloqueo sacroilíaco guiado por TC considerando exclusivamente aquellos con respuesta positiva. Se definió "respuesta positiva" a la reducción $>75 \%$ del puntaje de dolor prebloqueo según la escala analógica visual luego de la intervención por más de dos horas. ${ }^{10}$

Todos los pacientes con dolor sacroilíaco en nuestro centro reciben el mismo esquema diagnóstico y terapéutico en colaboración con el equipo de kinesiología. Ante un primer episodio de síntomas sacroilíacos positivos (palpación dolorosa, maniobras provocadoras de dolor positivas) se indica el tratamiento conservador:

1. Fase aguda (1-3 días): reposo, analgésicos no esteroides.

2. Fase de recuperación (de 3 días a 8 semanas): movilización articular, fisioterapia.

3. Fase de mantenimiento: cambios en la dinámica postural.

Los pacientes con síntomas persistentes luego de 10 sesiones son sometidos a un bloqueo sacroilíaco guiado por TC con fines diagnóstico y terapéutico. 


\section{Técnica del procedimiento}

Paciente en decúbito prono, se marcan reparos de anatomía superficial, línea media y cresta ilíaca. Se realiza la infiltración local de la piel con lidocaína al 1\%, se ingresa con aguja espinal 22G en la articulación sacroilíaca corroborando con cortes tomográficos como se muestra en la Figura 1. Se infiltran $1 \mathrm{ml}$ de bupivacaína al 0,5\% (40 mg), $2 \mathrm{ml}$ de lidocaína y $3 \mathrm{ml}$ de triamcinolona.

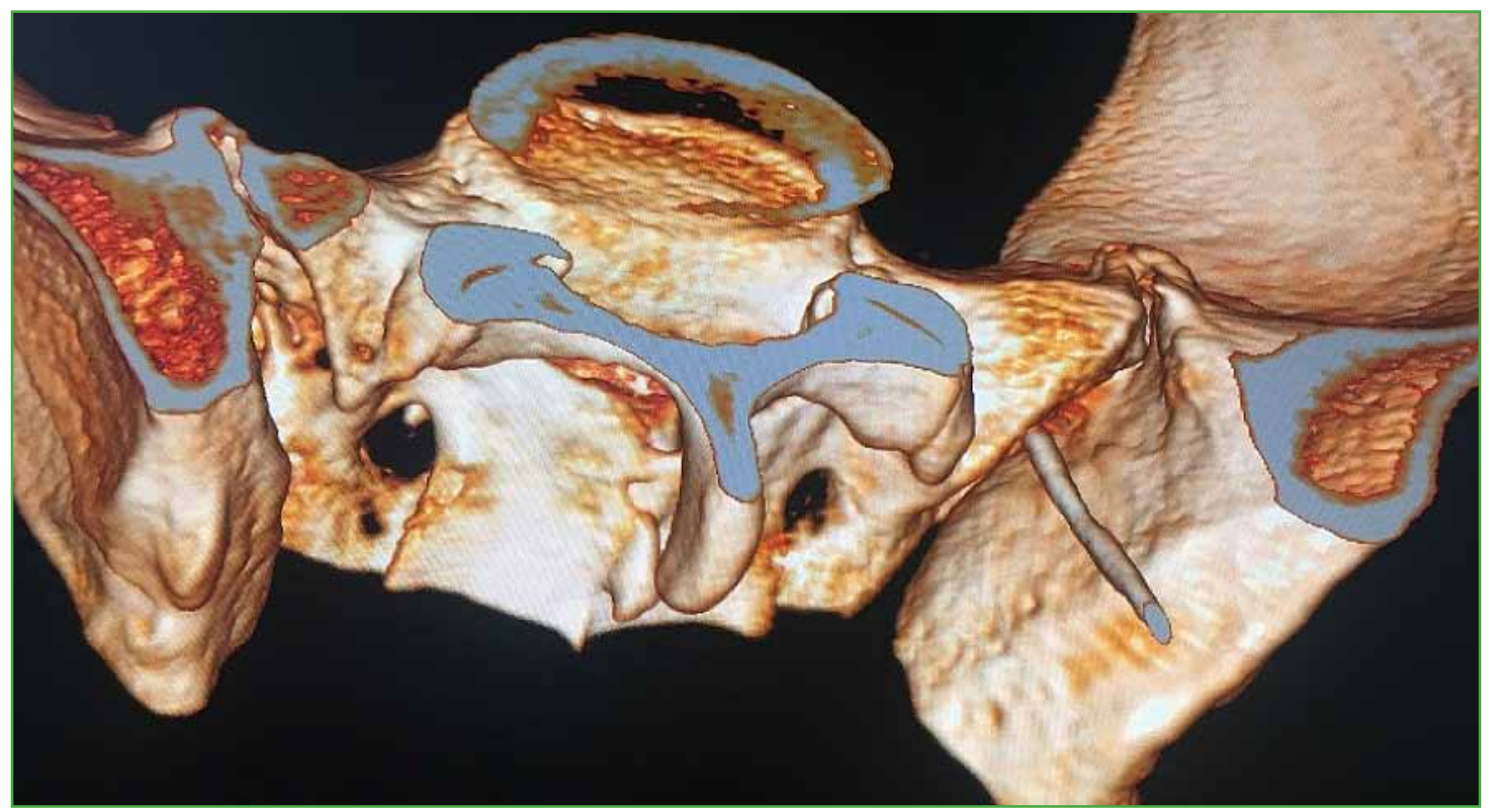

Figura 1. Imagen de reconstrucción de tomografía computarizada multiplanar.

\section{Variables de estudio}

Se registraron las siguientes variables del archivo de historias clínicas e imágenes:

Variables demográficas: edad y sexo.

Variables radiográficas:

- IP (ángulo entre el centro de rotación de ambas caderas y la perpendicular al centro del platillo superior del sacro) (Figura 2). 5

- Clasificación radiográfica de patología sacroilíaca según los criterios modificados de Nueva York: grado $0=$ normal, grado 1 = imagen dudosa de inflamación sacroilíaca, grado $2=$ esclerosis o cambios degenerativos localizados, sin compromiso del espacio articular, grado 3 = compromiso avanzado, esclerosis, geodas, disminución del espacio articular, puentes óseos, grado 4 = anquilosis sacroilíaca. ${ }^{11}$

Variables clínicas:

- Antecedente traumático: trauma (directo o indirecto), esfuerzos cíclicos (por repetición).

- Días de tratamiento posteriores al bloqueo sacroilíaco guiado por TC: esta variable numérica se configuró como variable nominal para su análisis en dos grupos según la presencia o la ausencia de síntomas (persistentes o recidivantes) a los 30 días del bloqueo.

Se seleccionó una muestra aleatoria de pacientes a través del programa SPSS Statics 25 sobre el total de pacientes, que fueron estudiados con TC lumbopélvica sin enfermedad de la pelvis o la columna lumbosacra evaluados durante el período de estudio, corroborando que no hubiera diferencias estadísticamente significativas respecto de la edad y el sexo.

Además, se dividió a los pacientes con dolor sacroilíaco en dos grupos, según los días de tratamiento posteriores al bloqueo sacroilíaco guiado por TC para comparar el valor de la IP en función de la presencia o ausencia de síntomas (persistentes o recidivantes) a los 30 días del bloqueo.

Las mediciones radiográficas se realizaron con el programa Surgimap for Windows Version 2.3.0. ${ }^{12}$ 


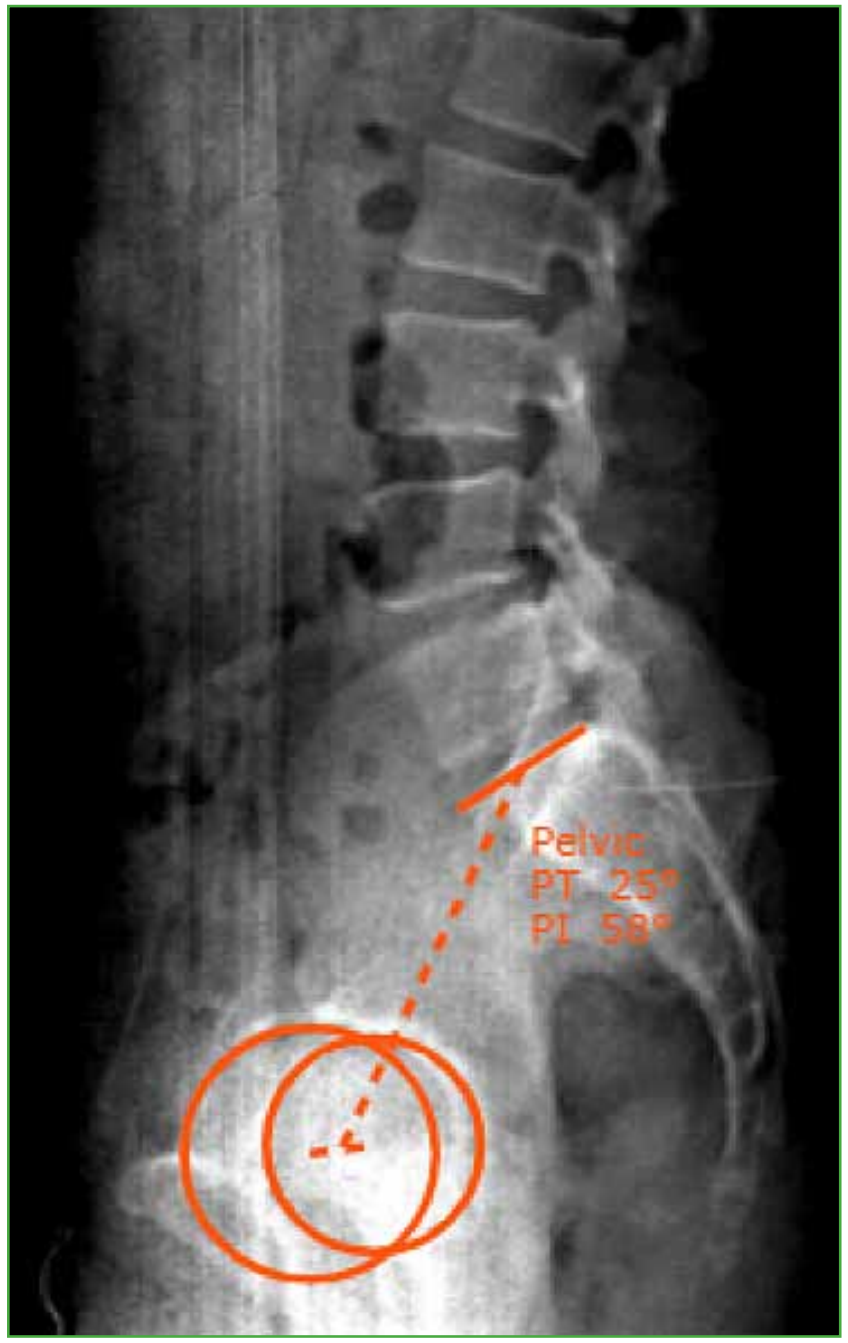

Figura 2. Medición radiográfica de la incidencia pélvica.

\section{Análisis estadístico}

Las variables categóricas se expresan en número y frecuencia relativa porcentual, y se analizaron con las pruebas de ji al cuadrado o de Fisher. Las variables interválicas se describen con media y su medida de dispersión (desviación estándar, DE). Para la comparación de las variables continuas se utilizaron la prueba t de Student o de suma de rangos de Wilcoxon, de acuerdo con la distribución expresada. Se consideró estadísticamente significativo un valor $\mathrm{p}<0,05$. Se utilizó el programa SPSS Statics 25 para el análisis.

\section{RESULTADOS}

Entre enero de 2015 y diciembre de 2018, se realizaron 830 TC de la región lumbopélvica. Sesenta y seis pacientes tenían una TC como guía de bloqueos analgésicos sacroilíacos; de este último grupo, 45 fueron excluidos por fusión lumbosacra (5 casos), uso de ortesis toracolumbar (4 casos), fractura de pelvis previa (10 casos), osteosíntesis sacroilíaca (10 casos), estudios incompletos ( 5 casos), bloqueos repetidos (6 casos) y respuesta negativa (5 casos), por lo que se conformó un grupo de 21 pacientes.

De 764 personas con TC de columna lumbar y pelvis sin enfermedad en ambas regiones, se excluyó a 20, porque no tenían escanograma de perfil, y a 30, porque no se visualizaban ambas caderas completas en el estudio. 
Finalmente, de esta población, se seleccionó una muestra aleatoria exacta de 21 pacientes como grupo de comparación.

No había diferencias estadísticamente significativas con respecto a la edad (p 0,330) y el sexo (p 1,000) entre ambos grupos. La IP media en pacientes con dolor sacroilíaco fue 52,57 (DE 9) y 46,52 (DE 9) en el otro grupo. La diferencia fue estadísticamente significativa con valores mayores de IP en pacientes con dolor sacroilíaco ( $p$ 0,042). Los resultados se resumen en la Tabla 1. No se obtuvieron diferencias significativas en la edad, el sexo, el antecedente traumático y la clasificación radiográfica de la ASI entre pacientes con síntomas que duraron $\leq 30$ días posbloqueo y aquellos con persistencia más allá de los 30 días (Tabla 2).

En la Tabla 3, se detallan los datos individuales (edad, sexo y valor de IP) de los pacientes con dolor sacroilíaco y los del grupo de comparación incluidos en el análisis.

Tabla 1. Variables en función del diagnóstico

\begin{tabular}{|l|c|c|c|}
\hline & $\begin{array}{c}\text { Pacientes con dolor sacroilíaco } \\
(\mathbf{n}=\mathbf{2 1})\end{array}$ & $\begin{array}{c}\text { Personas sanas } \\
(\mathbf{n}=\mathbf{2 1})\end{array}$ \\
\hline $\begin{array}{l}\text { Edad, media (DE) } \\
\text { IC95\% }\end{array}$ & $34(8)$ & $37(10)$ & 0,330 \\
\hline $\begin{array}{l}\text { Sexo, } \mathbf{n}(\%) \\
\text { Masculino }\end{array}$ & $30-38$ & $32-42$ & \\
\hline Femenino & $13(61,9)$ & $14(66,7)$ & 1,000 \\
\hline Incidencia pélvica, media (DE) & $8(38,1)$ & $7(33,3)$ & \\
\hline IC95\% & $52,57(9)$ & $46,52(9)$ & $\mathbf{0 , 0 4 2}$ \\
\hline
\end{tabular}

$\mathrm{DE}=$ desviación estándar

Tabla 2. Variables en función de duración de los síntomas

\begin{tabular}{|c|c|c|c|}
\hline & $\begin{array}{l}\leq 30 \text { días } \\
(n=9)\end{array}$ & $\begin{array}{l}>30 \text { días } \\
(\mathrm{n}=12)\end{array}$ & $\mathbf{p}$ \\
\hline $\begin{array}{l}\text { Edad, media (DE) } \\
\text { IC95\% }\end{array}$ & $\begin{array}{l}31(9) \\
25-38\end{array}$ & $\begin{array}{l}36(8) \\
31-40\end{array}$ & 0,229 \\
\hline $\begin{array}{c}\text { Sexo, n (\%) } \\
\text { Masculino } \\
\text { Femenino }\end{array}$ & $\begin{array}{l}7(78) \\
2(22)\end{array}$ & $\begin{array}{l}6(50) \\
6(50)\end{array}$ & 0,367 \\
\hline $\begin{array}{l}\text { Incidencia pélvica, media (DE) } \\
\text { IC95\% }\end{array}$ & $\begin{array}{l}53(9) \\
45-59\end{array}$ & $\begin{array}{l}53(9) \\
47-58\end{array}$ & 0,956 \\
\hline $\begin{array}{l}\text { Clasificación de Nueva York, n (\%) } \\
\text { Grado 0-1 } \\
\text { Grado } 2\end{array}$ & $\begin{array}{l}7(78) \\
2(22)\end{array}$ & $\begin{array}{l}8(67) \\
4(33)\end{array}$ & 0,659 \\
\hline $\begin{array}{l}\text { Trauma, n (\%) } \\
\text { Trauma (directo o indirecto) } \\
\text { Esfuerzos cíclicos }\end{array}$ & $\begin{array}{l}7(78) \\
2(22)\end{array}$ & $\begin{array}{l}10(83) \\
2(17)\end{array}$ & 1,000 \\
\hline
\end{tabular}

$\mathrm{DE}=$ desviación estándar. 
Tabla 3. Datos individuales de pacientes con dolor sacroilíaco y asintomáticos

\begin{tabular}{|c|c|c|c|c|}
\hline $\mathbf{n}$ & Grupo & Edad & Sexo & Incidencia pélvica \\
\hline 1 & Dolor sacroilíaco & 24 & M & 38 \\
\hline 2 & Dolor sacroilíaco & 37 & M & 42 \\
\hline 3 & Dolor sacroilíaco & 29 & $\mathrm{~F}$ & 44 \\
\hline 4 & Dolor sacroilíaco & 42 & M & 44 \\
\hline 5 & Dolor sacroilíaco & 19 & M & 45 \\
\hline 6 & Dolor sacroilíaco & 44 & M & 46 \\
\hline 7 & Dolor sacroilíaco & 48 & M & 47 \\
\hline 8 & Dolor sacroilíaco & 39 & M & 47 \\
\hline 9 & Dolor sacroilíaco & 23 & $\mathrm{~F}$ & 47 \\
\hline 10 & Dolor sacroilíaco & 29 & $\mathrm{~F}$ & 49 \\
\hline 11 & Dolor sacroilíaco & 28 & $\mathrm{~F}$ & 51 \\
\hline 12 & Dolor sacroilíaco & 31 & M & 51 \\
\hline 13 & Dolor sacroilíaco & 32 & M & 54 \\
\hline 14 & Dolor sacroilíaco & 34 & $\mathrm{~F}$ & 58 \\
\hline 15 & Dolor sacroilíaco & 48 & M & 58 \\
\hline 16 & Dolor sacroilíaco & 38 & $\mathrm{~F}$ & 58 \\
\hline 17 & Dolor sacroilíaco & 25 & M & 61 \\
\hline 18 & Dolor sacroilíaco & 32 & M & 61 \\
\hline 19 & Dolor sacroilíaco & 37 & M & 65 \\
\hline 20 & Dolor sacroilíaco & 44 & $\mathrm{~F}$ & 65 \\
\hline 21 & Dolor sacroilíaco & 33 & $\mathrm{~F}$ & 73 \\
\hline 22 & Asintomático & 44 & M & 28 \\
\hline 23 & Asintomático & 29 & M & 29 \\
\hline 24 & Asintomático & 26 & $\mathrm{~F}$ & 29 \\
\hline 25 & Asintomático & 39 & M & 39 \\
\hline 26 & Asintomático & 36 & $\mathrm{~F}$ & 40 \\
\hline 27 & Asintomático & 31 & $\mathrm{~F}$ & 42 \\
\hline 28 & Asintomático & 23 & $\mathrm{~F}$ & 43 \\
\hline 29 & Asintomático & 32 & $\mathrm{~F}$ & 43 \\
\hline 30 & Asintomático & 47 & M & 46 \\
\hline 31 & Asintomático & 59 & M & 48 \\
\hline 32 & Asintomático & 49 & M & 48 \\
\hline 33 & Asintomático & 43 & M & 48 \\
\hline 34 & Asintomático & 30 & M & 49 \\
\hline 35 & Asintomático & 47 & M & 52 \\
\hline 36 & Asintomático & 56 & M & 53 \\
\hline 37 & Asintomático & 40 & $\mathrm{~F}$ & 55 \\
\hline 38 & Asintomático & 45 & M & 55 \\
\hline 39 & Asintomático & 23 & M & 56 \\
\hline 40 & Asintomático & 26 & M & 57 \\
\hline 41 & Asintomático & 30 & M & 57 \\
\hline 42 & Asintomático & 23 & $\mathrm{~F}$ & 60 \\
\hline
\end{tabular}

$\mathrm{M}=$ masculino, $\mathrm{F}=$ femenino. 


\section{DISCUSIÓN}

La ASI es una causa común de dolor lumbosacro, especialmente en pacientes con actividades de carga repetitiva y asimétrica. ${ }^{13}$ El diagnóstico definitivo requiere un abordaje minucioso para diferenciarlo de otras topografías dolorosas, como columna lumbar y cadera, dolores irradiados a la región glútea y, en especial, enfermedades que pueden comprometer la ASI, como espondilitis anquilosante, enfermedad inflamatoria intestinal, artropatía psoriásica, infecciones, tumores, trastornos metabólicos, enfermedad degenerativa, afecciones iatrogénicas y colagenopatías., ${ }^{3,14}$

Varios factores, como la enfermedad degenerativa, la laxitud articular y el trauma, se han atribuido a su etiología. Nagamoto y cols. evaluaron la movilidad articular sacroilíaca en pacientes con enfermedad lumbar degenerativa y observaron que el movimiento articular en este grupo era significativamente mayor que en voluntarios sanos. ${ }^{15}$ Esto podría relacionarse con la susceptibilidad de estos pacientes al desarrollo de dolor sacroilíaco. ${ }^{16}$ En nuestro estudio, decidimos controlar estas variables excluyendo aquellos pacientes con enfermedad lumbosacra previa, fusión y ortesis.

En un estudio de la cinemática articular en pacientes con dolor sacroilíaco y controles sanos, Adhia y cols. observaron variaciones en la biomecánica articular. ${ }^{17}$

En las últimas décadas, múltiples autores han demostrado el valor del estudio de la anatomía sagital de la pelvis y la columna vertebral. ${ }^{4-9}$ La IP y los parámetros espinopélvicos se han evaluado en múltiples enfermedades del raquis, como la espondilolistesis y las deformidades vertebrales. Asimismo, se ha determinado la importancia de su evaluación en la planificación de cirugías de reemplazo articular de cadera y síndromes de fricción femoroacetabular. Cho y cols. estudiaron la morfología sacropélvica sagital y el balance espinopélvico en pacientes con dolor sacroilíaco durante el posoperatorio de cirugías de fusión lumbar. No encontraron diferencias en la medición de la IP entre pacientes con dolor sacroilíaco y los asintomáticos. Sí obtuvieron diferencias estadísticamente significativas en el aumento de la retroversión pélvica, en el posoperatorio de pacientes con síntomas sacroilíacos. ${ }^{18}$ En nuestro estudio, no medimos parámetros espinopélvicos que estiman el grado de retroversión pélvica, como la inclinación pélvica o la pendiente sacra, por tratarse de mediciones en TC en decúbito dorsal.

Nuestro estudio representa una inquietud no expuesta aún en la bibliografía actual al estudiar la morfología sagital de la región sacropélvica de pacientes con dolor sacroilíaco. No obtuvimos diferencias entre la media de IP de nuestra población y los valores que se proponen en la bibliografía. ${ }^{5,6}$ Sin embargo, el valor de IP significativamente mayor en pacientes con dolor sacroilíaco que en los asintomáticos (p 0,046) plantea la necesidad futura de profundizar en el estudio de los parámetros espinopélvicos de estos pacientes.

Las debilidades de nuestro estudio se asocian a su carácter retrospectivo y la escasa cantidad de pacientes. En detrimento de la potencia estadística del estudio, decidimos controlar variables que podrían operar como factores de confusión excluyendo a pacientes con enfermedades previas de la pelvis y la columna vertebral, múltiples siniestros laborales y trauma de pelvis de alta energía.

Consideramos relevante llevar a cabo estudios con un mayor nivel de evidencia que permitan estimar la relación de la IP y el riesgo de dolor sacroilíaco.

\section{CONCLUSIÓN}

Los pacientes con dolor sacroilíaco tuvieron una IP mayor que las personas sanas, con una diferencia estadísticamente significativa en nuestro estudio. No obtuvimos diferencias en función de la duración de los síntomas. Consideramos interesante nuestro aporte sobre la anatomía sagital de la pelvis en pacientes con dolor sacroilíaco, porque brinda información sin antecedentes en la bibliografía y aporta consideraciones para el desarrollo de nuevas hipótesis en la génesis del dolor sacroilíaco.

Conflicto de intereses: Los autores no declaran conflictos de intereses. 


\section{BIBLIOGRAFÍA}

1. Cohen SP. Sacroiliac joint pain: a comprehensive review of anatomy, diagnosis, and treatment. Anesth Analg 2005;101(5):1440-53. https://doi.org/10.1213/01.ANE.0000180831.60169.EA

2. Schmidt GL, Bhandutia AK, Altman DT. Management of sacroiliac joint pain. J Am Acad Orthop Surg 2018;26(17):610-6. https://doi.org/10.5435/JAAOS-D-15-00063

3. Thawrani DP, Agabegi SS, Asghar F. Diagnosing sacroiliac joint pain. J Am Acad Orthop Surg 2019;27(3):85-93. https://doi.org/10.5435/JAAOS-D-17-00132

4. Duval-Beaupère G, Schmidt C, Cosson P. A barycentremetric study of the sagittal shape of spine and pelvis: the conditions required for an economic standing position. Ann Biomed Eng 1992;20(4):451-62. https://doi.org/10.1007/BF02368136

5. Legaye J, Duval-Beaupère G, Hecquet J, Marty C. Pelvic incidence: a fundamental pelvic parameter for threedimensional regulation of spinal sagittal curves. Eur Spine J 1998;7(2):99-103.

https://doi.org/10.1007/s005860050038

6. Vialle R, Levassor N, Rillardon L, Templier A, Skalli W, Guigui P. Radiographic analysis of the sagittal alignment and balance of the spine in asymptomatic subjects. J Bone Joint Surg Am 2005;87(2):260-7. https://doi.org/10.2106/JBJS.D.02043

7. Le Huec JC, Aunoble S, Philippe L, Nicolas P. Pelvic parameters: origin and significance. Eur Spine J 2011;20 Suppl 5(Suppl 5):564-71. https://doi.org/10.1007/s00586-011-1940-1

8. Schwab F, Lafage V, Boyce R, Skalli W, Farcy JP. Gravity line analysis in adult volunteers: age-related correlation with spinal parameters, pelvic parameters, and foot position. Spine (Phila Pa 1976) 2006;31(25):E959-67. https://doi.org/10.1097/01.brs.0000248126.96737.0f

9. Schwab F, Lafage V, Patel A, Farcy JP. Sagittal plane considerations and the pelvis in the adult patient. Spine (Phila Pa 1976) 2009;34(17):1828-33. https://doi.org/10.1097/BRS.0b013e3181a13c08

10. Mitchell B, McPhail T, Vivian D, Verrills P, Barnard A. Diagnostic sacroiliac joint injections: Is a control block necessary? J Sci Med Sport 2010;12:e5-e6. https://doi.org/10.1016/j.jsams.2009.10.012

11. Van der Linden S, Valkenburg HA, Cats A. Evaluation of diagnostic criteria for ankylosing spondylitis. A proposal for modification of the New York criteria. Arthritis Rheum 1984;27(4):361-8. https://doi.org/10.1002/art.1780270401

12. Lafage R, Ferrero E, Henry JK, Challier V, Diebo B, Liabaud B, et al. Validation of a new computer-assisted tool to measure spino-pelvic parameters. Spine J 2015;15(12):2493-502. https://doi.org/10.1016/j.spinee.2015.08.067

13. Peebles R, Jonas CE. Sacroiliac joint dysfunction in the athlete: diagnosis and management. Curr Sports Med Rep 2017;16(5):336-42. https://doi.org/10.1249/JSR.0000000000000410

14. Zelle BA, Gruen GS, Brown S, George S. Sacroiliac joint dysfunction: evaluation and management. Clin J Pain 2005;21(5):446-55. https://doi.org/10.1097/01.ajp.0000131413.07468.8e

15. Nagamoto Y, Iwasaki M, Sakaura H, Sugiura T, Fujimori T, Matsuo Y, et al. Sacroiliac joint motion in patients with degenerative lumbar spine disorders. J Neurosurg Spine 2015;23(2):209-16. https://doi.org/10.3171/2014.12.SPINE14590

16. Yoshihara H. Sacroiliac joint pain after lumbar/lumbosacral fusion: current knowledge. Eur Spine J 2012;21(9): 1788-96. https://doi.org/10.1007/s00586-012-2350-8

17. Adhia DB, Milosavljevic S, Tumilty S, Bussey MD. Innominate movement patterns, rotation trends and range of motion in individuals with low back pain of sacroiliac joint origin. Man Ther 2016;21:100-8. https://doi.org/10.1016/j.math.2015.06.004

18. Cho DY, Shin MH, Hur JW, Ryu KS, Park CK. Sagittal sacropelvic morphology and balance in patients with sacroiliac joint pain following lumbar fusion surgery. J Korean Neurosurg Soc 2013;54(3):201-6.

https://doi.org/10.3340/jkns.2013.54.3.201 